\title{
On the Transition from Ferromagnetism to Antiferromagnetism on Twofold Cayley Tree.
}

\author{
Can F. Delale \\ Department of Mathematics, Bilkent University - 06533 Bilkent, Ankara, Turkey
}

(ricevuto il 26 Aprile 1991; revisionato il 18 Ottobre 1991; approvato il 9 Dicembre 1991)

\begin{abstract}
Summary. - A fixed-point conversion theorem which shows the transition from ferromagnetism to antiferromagnetism on twofold Cayley tree is proved. The ferromagnetic and antiferromagnetic maps are shown to be related by an involution and in zero field the stable fixed points of the ferromagnetic map are converted to a stable two-cycle of the antiferromagnetic map. A reduced one-dimensional analysis in zero field yields precisely the same results.

PACS 64.60.Cn - Order-disorder and statistical mechanics of model systems.

PACS $64.70 . \mathrm{Kb}$ - Solid-solid transitions.

PACS 05.50 - Lattice theory and statistics; Ising problems.
\end{abstract}

\section{1. - Introduction.}

Spin systems on hierarchical graphs have been of considerable interest in recent years because many statistical properties of these systems can be obtained exactly [1]. The simplest of these is the Ising model on a Cayley tree whose exact solution [2-6] exhibits very interesting features. It is well known that the classical Bethe-Peierls equations cannot be produced exactly on this graph, but only when surface effects are eliminated the Bethe-Peierls transition can occur. An approach [7] to produce the Bethe-Peierls transition on the Cayley tree in the accepted statistical mechanical fashion is to apply appropriate boundary fields which are determined by the Bethe-Peierls equations. An extension of this method to a mean field spin glass theory is also available [8]. Another approach taken by Jelitto[9] in joining two identical Cayley trees at their boundaries leads to a different-type transition. An improvement on this approach is taken by the present author [10] by constructing a two-fold Cayley tree which shares the $q$ coordination property of a Bethe lattice. The ferromagnetic Ising model on this graph is solved exactly and a phase transition in zero field at the critical Bethe temperature is found. Although the transition is similar in nature to the Bethe-Peierls transition and occurs at the same critical 
temperature, it differs from the Bethe-Peierls transition in that all sites on the twofold Cayley tree graph are not topologically equivalent owing to the existence of closed loops which are absent on a Bethe lattice.

This study extends the ferromagnetic solution [10] on twofold Cayley tree to the corresponding antiferromagnetic solution by inverting the control parameter which characterizes the temperature of the lattice. The maps characterizing the ferromagnetic and antiferromagnetic systems are shown to be related by an involution which characterizes the transition in general. A fixed-point conversion theorem which shows how in zero field the stable fixed points of the ferromagnetic system are converted to a stable two-cycle of the antiferromagnetic system is proved. The reduced one-dimensional analysis of this transition is worked out in detail and is found in agreement with the fixed-point conversion theorem of this investigation.

\section{2. - Transition from ferromagnetism to antiferromagnetism on twofold Cayley tree.}

In this section we discuss how the solution of the ferromagnetic Ising model on a twofold Cayley tree can be related to the antiferromagnetic solution on the same tree. A twofold Cayley tree graph is constructed by joining two open Cayley trees each containing $n-1$ shells at their boundaries by adding small polygons to ensure that all sites have the same coordination number $q$. A typical twofold Cayley tree graph for $n=3$ and $q=3$ is shown in fig. 1. The Ising model on this graph can be solved exactly[10] and shows a phase transition in zero field at the critical Bethe temperature. The magnetization $M$ for sites far removed from the boundary can be explicitly evaluated by

$$
M=\frac{\beta^{2}-x^{q}}{\beta^{2}+2 \beta y^{q}+x^{q}},
$$

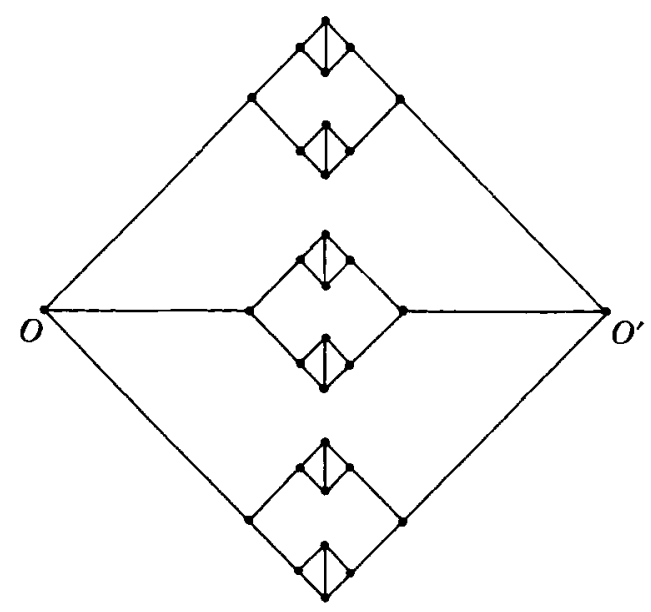

Fig. 1. - A twofold Cayley tree with $n=q=3$. 
where $x$ and $y$ satisfy the discrete two-dimensional dynamical system

$$
\left\{\begin{array}{l}
x=f(x, y ; \alpha, \beta)=\frac{\beta^{2}+2 \alpha \beta y^{q-1}+\alpha^{2} x^{q-1}}{\alpha^{2} \beta^{2}+2 \alpha \beta y^{q-1}+x^{q-1}}, \\
y=g(x, y ; \alpha, \beta)=\frac{\alpha \beta^{2}+\beta\left(\alpha^{2}+1\right) y^{q-1}+\alpha x^{q-1}}{\alpha^{2} \beta^{2}+2 \alpha \beta y^{q-1}+x^{q-1}} .
\end{array}\right.
$$

In eqs. (1) and (2), $\alpha \equiv \exp [2 \varepsilon / k T]$ and $\beta \equiv \exp [2 B / k T]$, where $\varepsilon$ is the nearestneighbour interaction constant, $B$ is the external magnetic field, $k$ is Boltzmann's constant and $T$ is the temperature of the lattice.

When $\alpha>1(\varepsilon>0)$, eq. (1) represents the magnetization for sites deep within the graph for the ferromagnetic phase since all such sites are equivalent. However, in the antiferromagnetic case where $0<\alpha<1(\varepsilon<0)$ the local magnetization for sites far removed from the boundary alternate between neighbouring shells since as is well known only sites located on the same shell away from the boundary have the same magnetization and sites deep within the graph on neighbouring shells have opposite magnetizations. In addition in the antiferromagnetic case there is frustration at the joining boundary of the two Cayley trees. We herein discuss how the transition between these two cases (ferromagnetic and antiferromagnetic) occurs in general.

We let $J \equiv(1, \infty)$ and from now on $\alpha \in J$ (i.e. from now on we define $\alpha$ by $\alpha \equiv \exp [2|\varepsilon| / k T])$. Further we define $z \equiv(x, y) \in \mathfrak{R}^{+2}, \quad \gamma \equiv(\alpha, \beta) \in J \times \mathfrak{R}^{+}$and $h \equiv(f, g)$. Then the ferromagnetic model described by the dynamical system (2) reads

$$
z=h(z, \gamma) .
$$

If we now let $\hat{\gamma} \equiv(1 / \alpha, \beta)$ and define

$$
\left\{\begin{array}{l}
F(z, \gamma)=f(z, \hat{\gamma})=\frac{\alpha^{2} \beta^{2}+2 \alpha \beta y^{q-1}+x^{q-1}}{\beta^{2}+2 \alpha \beta y^{q-1}+\alpha^{2} x^{q-1}}, \\
G(z, \gamma) \equiv g(z, \hat{\gamma})=\frac{\alpha \beta^{2}+\beta\left(\alpha^{2}+1\right) y^{q-1}+\alpha x^{q-1}}{\beta^{2}+2 \alpha \beta y^{q-1}+\alpha^{2} x^{q-1}}
\end{array}\right.
$$

and $H \equiv(F, G)$, the two-dimensional dynamical system

$$
z=H(z, \gamma)
$$

then describes the solution for the antiferromagnetic model on the twofold Cayley tree.

In what follows we show how a solution of the ferromagnetic system (3) is converted to a solution of the antiferromagnetic system (5) describing the transition in general.

Proposition 2.1. The ferromagnetic map $h \equiv(f, g)$ and the antiferromagnetic map $H \equiv(F, G)$ are related by the transformation

$$
F(z, \gamma)=\frac{1}{f(z, \gamma)}
$$


and

$$
G(z, \gamma)=\frac{g(z, \gamma)}{f(z, \gamma)}
$$

Proof. Obvious by eqs. (2) and (4).

Proposition 2.2. Let $\bar{z} \equiv(1 / x, y / x)$ and $\bar{\gamma} \equiv(\alpha, 1 / \beta)$. Then $H(\bar{z}, \bar{\gamma})=h(z, \gamma)$ and $h(\bar{z}, \bar{\gamma})=H(z, \gamma)$.

Proof. Let $\Lambda \equiv J \times \Re^{+}$and define the involution $j: \Re^{+2} \times \Lambda \rightarrow \mathfrak{R}^{+2} \times \Lambda$ by $j(z, \gamma)=$ $=(\bar{z}, \bar{\gamma})$. Also let $j \bar{f} \equiv \bar{f} \circ j$ for any map $\widehat{f}$ whose domain is $\mathfrak{R}^{+2} \times \Lambda$. We then have

$$
j h(z, \gamma)=h \circ j(z, \gamma)=h(\bar{z}, \bar{\gamma})=(f(\bar{z}, \bar{\gamma}), g(\bar{z}, \bar{\gamma})) .
$$

On the other hand, straightforward evaluation yields $f(\bar{z}, \bar{\gamma})=1 / f(z, \gamma)$ and $g(\bar{z}, \bar{\gamma})=$ $=g(z, \gamma) / f(z, \gamma)$. In virtue of proposition 2.1, we obtain the result $j h=H$. Since $j$ is an involution on $\mathfrak{R}^{+2} \times A\left(j^{2}=\right.$ identity), the result $j H=h$ follows which proves the proposition.

Theorem 2.3 (Fixed-point conversion theorem). Let $z$ be a fixed point of $h$ for some $\gamma$. Then $\bar{z}$ is a fixed point of $h$ for $\bar{\gamma}$ (or equivalently $\bar{z}=H(z, \gamma)$ ) $\left(^{*}\right)$. In particular, when $\gamma=\bar{\gamma}=\gamma_{0} \equiv(\alpha, 1)$, i.e. in zero field, $\bar{z}$ is a fixed point of $h$ for $\gamma_{0}$ and the fixed points $(z, \bar{z})$ of the ferromagnetic map $h$ are converted to a two-cycle of the antiferromagnetic map $H$, i.e.

$$
\bar{z}=H\left(z, \gamma_{0}\right) \quad \text { and } \quad z=H\left(\bar{z}, \gamma_{0}\right)
$$

whenever $z \neq \bar{z}$.

Proof. Let $p: \mathfrak{R}^{+2} \times \Lambda \rightarrow \mathfrak{R}^{+2}$ be given by $p(z, \gamma)=z$ and $z$ be a fixed point of $h$ for some $\gamma$. Then $p(z, \gamma)=h(z, \gamma)$. By operating $j$, we obtain

$$
j p(z, \gamma)=p(\bar{z}, \bar{\gamma})=\bar{z}=j h(z, \gamma)=h(\bar{z}, \bar{\gamma}),
$$

which shows that $\bar{z}$ is a fixed point of $h$ for $\bar{\gamma}$. By proposition 2.2 we have equivalently $\bar{z}=H(z, \gamma)$ and $z=H(\bar{z}, \bar{\gamma})$. When $\gamma=\bar{\gamma}=\gamma_{0} \equiv(\alpha, 1)$, we trivially obtain

$$
\bar{z}=h\left(\bar{z}, \gamma_{0}\right)
$$

and

$$
\bar{z}=H\left(z, \gamma_{0}\right)=h\left(z, \hat{\gamma}_{0}\right) \quad \text { and } \quad z=H\left(\bar{z}, \gamma_{0}\right)=h\left(\bar{z}, \hat{\gamma}_{0}\right),
$$

where $\hat{\gamma}_{0} \equiv(1 / \alpha, 1)$ as required.

Corollary 2.4. The point $z=1 \equiv(1,1)$ is a fixed point of both $h$ and $H$ for $\gamma_{0} \equiv(\alpha, 1)$. This fixed point is asymptotically stable whenever $1<\alpha<\alpha_{\mathrm{c}}$ (the

(*) This statement may also be written as «If $z$ is a fixed point of $h$ for some $\gamma$, then $z$ is a fixed point of $H_{1}$ for the same $\gamma$ where $H_{1} \equiv\left(F_{1}, G_{1}\right)$ with $F_{1}(z, \gamma) \equiv x^{2} F(z, \gamma)$ and $G_{1}(z, \gamma) \equiv x G(z, \gamma)$." 
paramagnetic phase) and unstable for $\alpha>\alpha_{c}$ (the ferromagnetic and antiferromagnetic phases) where $\alpha_{\mathrm{c}} \equiv q /(q-2), q>2$.

Proof. Consider the case where the involution $j$ in the proof of proposition 2.2 is the identity, i.e. the case when $z=\bar{z}=1 \equiv(1,1)$ and $\gamma=\bar{\gamma}=\gamma_{0}=(\alpha, 1)$. It then follows by theorem 2.3 that $z=1$ is a fixed point of both $h$ and $H$ for $\gamma_{0}$. To discuss the stability [11, 12] of the fixed point $z=1$ of $h$ and $H$, we consider the Jacobian matrices of $h$ and $H$ at $z=1$ and $\gamma=\gamma_{0}$; namely

$$
D h\left(1, \gamma_{0}\right)=\frac{q-1}{(\alpha+1)^{2}}\left[\begin{array}{cc}
\alpha^{2}-1 & 0 \\
\alpha-1 & (\alpha-1)^{2}
\end{array}\right]
$$

and

$$
D H\left(1, \gamma_{0}\right)=\frac{q-1}{(\alpha+1)^{2}}\left[\begin{array}{cc}
-\left(\alpha^{2}-1\right) & 0 \\
-\alpha(\alpha-1) & (\alpha-1)^{2}
\end{array}\right] \text {. }
$$

The eigenvalues $\lambda_{1,2}$ of $D h\left(1, \gamma_{0}\right)$ and $\bar{\lambda}_{1,2}$ of $D H\left(1, \gamma_{0}\right)$ then read

$$
\lambda_{1}=-\bar{\lambda}_{1}=(q-1) \frac{\alpha-1}{\alpha+1} \quad \text { and } \quad \lambda_{2}=\bar{\lambda}_{2}=(q-1)\left(\frac{\alpha-1}{\alpha+1}\right)^{2} .
$$

Since

$$
\left|\bar{\lambda}_{2}\right|=\left|\lambda_{2}\right|<\left|\bar{\lambda}_{1}\right|=\left|\lambda_{1}\right|=(q-1) \frac{\alpha-1}{\alpha+1}
$$

for $\alpha>1$, we have the fixed-point stability condition

$$
(q-1)\left(\frac{\alpha-1}{\alpha+1}\right)<1,
$$

which shows that for $\gamma_{0}$ the fixed point $z=1$ of both $h$ and $H$ is asymptotically stable when $1<\alpha<\alpha_{\mathrm{c}} \equiv q /(q-2)$ and unstable when $\alpha>\alpha_{\mathrm{c}}$ as required.

\section{3. - Analysis of zero-field results.}

In this section we analyse the results of zero field $(\beta=1)$ in some detail. In this case eqs. (2) for the ferromagnetic system take the form

$$
\left\{\begin{array}{l}
x=f(x, y ; \alpha, 1)=\frac{1+2 \alpha y^{q-1}+\alpha^{2} x^{q-1}}{\alpha^{2}+2 \alpha y^{q-1}+x^{q-1}}, \\
y=g(x, y ; \alpha, 1)=\frac{\alpha+\left(\alpha^{2}+1\right) y^{q-1}+\alpha x^{q-1}}{\alpha^{2}+2 \alpha y^{q-1}+x^{q-1}},
\end{array}\right.
$$


whereas eqs. (4) for the antiferromagnetic system reduce to

$$
\left\{\begin{array}{l}
x=F(x, y ; \alpha, 1)=\frac{\alpha^{2}+2 \alpha y^{q-1}+x^{q-1}}{1+2 \alpha y^{q-1}+\alpha^{2} x^{q-1}}, \\
y=G(x, y ; \alpha, 1)=\frac{\alpha+\left(\alpha^{2}+1\right) y^{q-1}+\alpha x^{q-1}}{1+2 \alpha y^{q-1}+\alpha^{2} x^{q-1}},
\end{array}\right.
$$

where $a>1$ for both systems. The magnetization per site $M$ for sites far removed from the boundary in turn becomes

$$
M=\frac{1-x^{q}}{1+2 y^{q}+x^{q}} .
$$

The analysis of the ferromagnetic system (6) has been carried out in great depth in ref. [10]. It is shown there that the system possesses the stable fixed point $(x=1, y=$ $=1$ ) for $\alpha<\alpha_{c}$ (the paramagnetic phase), while this point becomes unstable and the system bifurcates to the two stable fixed points $\left(x^{*}, y^{*}\right)$ and $\left(1 / x^{*}, y^{*} / x^{*}\right)$ for $\alpha>\alpha_{c}$ (the ferromagnetic phase), which can be readily observed from corollary 2.4 .

We herein focus our attention on the antiferromagnetic system (7). We already know that (7) possesses the fixed point $(x=1, y=1)$. The two-dimensional system (7) is in general difficult to solve; however, it can be reduced, after cumbersome manipulations, into the one-dimensional system

$$
x=K(x ; \alpha),
$$

where $K(x ; \alpha)$ is given by

$$
K(x ; \alpha)=1+\frac{\left(\alpha^{2}-1\right)\left(1-x^{q-1}\right)}{1+2 \alpha\left[\frac{x^{q-1}-x+\alpha^{2}\left(x^{q}-1\right)}{2 \alpha\left(x^{q-1}-1\right)}\right]^{q-1}+\alpha^{2} x^{q-1}}, \quad x \neq 1,
$$

which seems easier to analyse. Once the one-dimensional system (9) is solved, the variable $y$ can in turn be obtained from

$$
\begin{cases}y(x)=\frac{x^{q-1}-x+\alpha^{2}\left(x^{q}-1\right)}{2 \alpha\left(x^{q-1}-1\right)} & \text { for } x \neq 1, \\ y(1)=1 & \text { for } x=1 .\end{cases}
$$

The properties of the antiferromagnetic system can now be deduced from the analysis of the function $K$ for $x \in \Re^{+}$and $\alpha>1$. We first notice that $K(x ; \alpha)$ is continuous in $x$ on $\mathfrak{R}^{+}$except at $x=1$ where it exhibits a removable discontinuity. We can remove this discontinuity by defining

$$
K(1 ; \alpha)=1
$$

making $K$ continuous in $x$ on $\Re^{+}$for $\alpha>1$. Moreover, $K$ can be made differentiable in $x$ on $\mathfrak{R}^{+}$for $\alpha>1$. The derivative of $K$ with respect to $x$ at $x=1$ can then be 

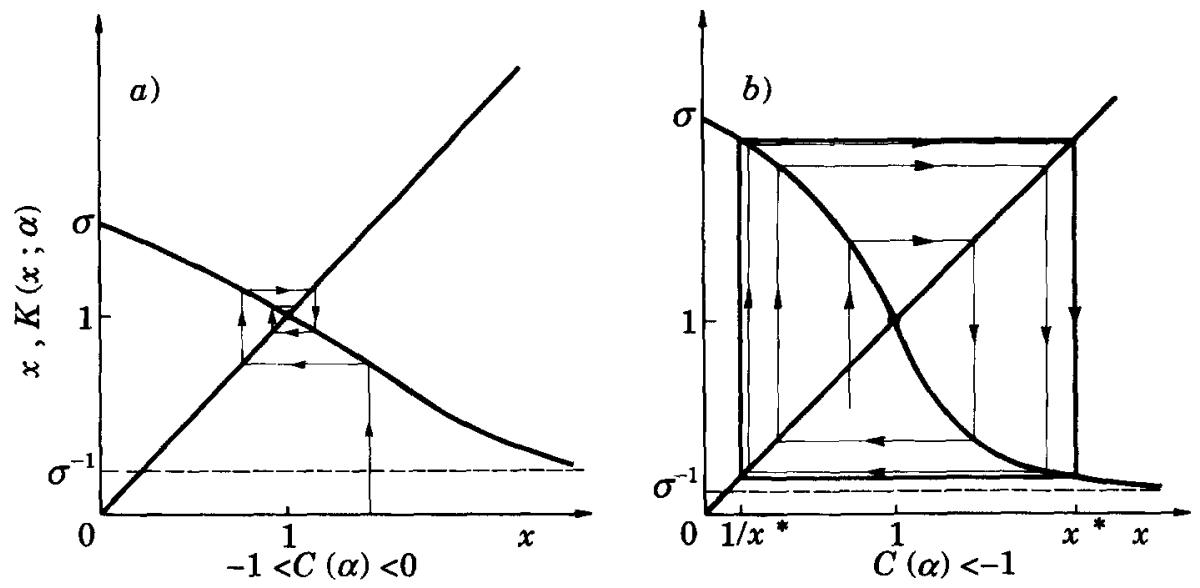

Fig. 2. - Graphical solution of the reduced one-dimensional system (9)

$$
\left[\sigma \equiv \frac{\alpha^{q}+2^{q-2} \alpha^{2}}{\alpha^{q}+2^{q-2}}\right] .
$$

evaluated by

$$
C(\alpha) \equiv K_{x}(1 ; \alpha)=-\frac{(q-1)\left(\alpha^{2}-1\right)}{1+2 \alpha\left[\frac{q-2+q \alpha^{2}}{2 \alpha(q-1)}\right]^{q-1}+\alpha^{2}}
$$

It can also be shown that $K(x ; \alpha)$ is a decreasing function of $x$ for $x>0$ and $\alpha>1$. In particular, we have

$$
\left\{\begin{array}{l}
K(0 ; \alpha)=\frac{a^{q}+2^{q-2} \alpha^{2}}{\alpha^{q}+2^{q-2}}>1, \\
K(1 ; \alpha)=1 \\
K(x ; \alpha) \sim \frac{\alpha^{q}+2^{q-2}}{\alpha^{q}+2^{q-2} \alpha^{2}}<1 \quad \text { as } x \rightarrow \infty
\end{array}\right.
$$

Typical behaviour of $K(x, \alpha)$ for some fixed $\alpha$ is shown in fig. $2 a)$ and $b$ ). We now investigate the dynamical behaviour of eq. (9) for $x>0$ and $\alpha>1$. It is obvious by eq. (11) that $x=1$ (in turn $y(1)=1$ ) is a fixed point of the system (9) for $\alpha>1$ in agreement with corollary 2.4. Actually since $K(x ; \alpha)$ is a decreasing function of $x$ for $x>0$ and $\alpha>1$, it follows immediately (e.g., see fig. $2 a$ ) and $b$ )) that $x=1$ is the only fixed point of the system (9). To discuss the stability of the fixed point $x=1$ of the system (9) the following lemma proves to be useful.

Lemma 3.1. For all $\alpha>1$ and $q>2$

$$
-(q-1) \frac{\alpha-1}{\alpha+1}<C(\alpha)<0 \text {. }
$$

Proof. That $C(\alpha)<0$ for $\alpha>1$ and $q>2$ is obvious from the defining relation (12). Therefore, we only need to prove the left-hand side of the inequality. We first note 
that for $\alpha>1$ and $q>2$

$$
\left[\frac{q-2+q \alpha^{2}}{2 \alpha(q-1)}\right]^{q-1}>1
$$

since

$$
\frac{q-2+q \alpha^{2}}{2 \alpha(q-1)}>\frac{(q-2)\left(\alpha^{2}+1\right)}{2 \alpha(q-1)}+\frac{1}{q-1}>\frac{q-2}{q-1}+\frac{1}{q-1}=1 .
$$

Thus we have

$$
C(\alpha)=-\frac{(q-1)\left(\alpha^{2}-1\right)}{1+2 \alpha\left[\frac{q-2+q \alpha^{2}}{2 \alpha(q-1)}\right]^{q-1}+\alpha^{2}}>-\frac{(q-1)\left(\alpha^{2}-1\right)}{(\alpha+1)^{2}}=-\frac{(q-1)(\alpha-1)}{\alpha+1}
$$

as required.

It is well known that $x=1$ is a stable fixed point of the system (9) if $-1<C(\alpha)<0$ and is unstable whenever $C(\alpha)<-1$. In virtue of the above lemma this means that the fixed point $x=1$ is asymptotically stable whenever $(q-1)(\alpha-1) /(\alpha+1)<1$ or $1<\alpha<\alpha_{\mathrm{c}} \equiv q /(q-2)$. Thus by eq. (8) we have only the paramagnetic phase when $\alpha<\alpha_{\mathrm{c}}$. The antiferromagnetic phase emerges when the fixed point $x=1$ becomes unstable, i.e. when $C(\alpha)<-1$, a situation which may arise when $\alpha>\alpha_{\mathrm{c}}$. In addition since $K(x, \alpha)$ is a decreasing function of $x$ and is bounded for $\alpha>1$, it is well known [6] that even and odd iterates of the iterated system corresponding to eq. (9) form bounded monotonic sequences which either converge to the same fixed point $x=1$ (when $\alpha<\alpha_{\mathrm{c}}$ ) or to a stable two-cycle with values $x^{*}$ and $1 / x^{*}$ (when $\alpha>\alpha_{\mathrm{c}}$ as is also evident from fig. $2 a$ ) and $b$ ). By eq. (8) the spontaneous magnetization for sites far removed from the boundary either vanishes (the paramagnetic phase) or takes the periodic values $M^{*}$ or $-M^{*}$, where

$$
M^{*}=\frac{1-x^{* q}}{1+2 y^{* q}+x^{* q}}
$$

with $y^{*}=y\left(x^{*}\right)$ (the antiferromagnetic phase). In particular for $\alpha>\alpha_{\mathrm{c}}$ the periodic points $x^{*}$ and $1 / x^{*}$ of eq. (9) can be shown to be precisely the fixed points of the reduced one-dimensional ferromagnetic system (eq. (5.3) in ref. [10]). This result is in complete agreement with theorem 2.4 and is a characterization of the transition from ferromagnetism to antiferromagnetism in zero field.

\section{4. - Conclusions.}

The transition from ferromagnetic to antiferromagnetic coupling on a twofold Cayley tree is treated as an involutive transformation of the ferromagnetic map into the antiferromagnetic map described by two-dimensional discrete dynamical systems. As a bonus a fixed-point conversion theorem which shows how in zero field the stable fixed points of the ferromagnetic map are converted into a stable two-cycle of the antiferromagnetic map is proved. The results of this investigation confirm those $[5,6]$ obtained in calculating local properties on a Cayley tree and 
illuminate the nature of transition from ferromagnetic to antiferromagnetic coupling.

\section{REFERENCES}

[1] M. Kaufman and R. G. Griffiths: Phys. Rev. B, 24, 496 (1981).

[2] L. K. RUNNELS: J. Math. Phys., 8, 2081 (1967).

[3] T. P. Eggarter: Phys. Rev. B, 9, 2989 (1974).

[4] E. MÜller-HaRtMann and J. ZitTaRTZ: Phys. Rev. Lett., 33, 893 (1974).

[5] R. J. BAXTER: Exactly Solved Models in Statistical Mechanics (Academic Press, 1982), Chapt. 4.

[6] C. J. Thompson: J. Stat. Phys., 27, 441 (1982).

[7] F. Peruggi, F. Di Liberto and G. Monroy: J. Phys. A, 16, 811 (1983).

[8] J. T. Chayes, L. Chayes, J. P. Sethna and D. J. Thouless: Commun. Math. Phys., 106, 41 (1986).

[9] R. J. JelitTo: Physica A, 99, 268 (1979).

[10] C. F. Delale: Int. J. Mod. Phys. B, 3, 1523 (1989).

[11] J. M. T. Thompson and H. B. STEWART: Nonlinear Dynamics and Chaos (Wiley, 1986), p. 135.

[12] R. L. Devaney: An Introduction to Chaotic Dynamical Systems (Addison-Wesley, 1989). 